\title{
Improved Classification of Fetal Abnormalities Using Automated ABC ANFIS Classifier
}

\author{
Nagu Malothu, V.V.K.D.V. Prasad
}

\begin{abstract}
Early determination of fetal irregularities can be performed utilizing a legitimate screening technique. The screening may at some point look as a thorough one for therapeutic specialists. Thus, mechanization with manual investigation gives better help to endoscopist in discovering the strange fetal pictures. In this paper, we consider a robotized order of fetal irregularities amid first trimester pregnancy period utilizing Artificial Bee Colony (ABC) and Hybrid ANFIS. At first, the picture is pre-prepared to expel the clamor and other appearance exhibit in crude picture dataset. In the second stage, an $A B C$ calculation is utilized to section the picture into marks in light of district-based division. In the last stage, the picture names are grouped utilizing half and half ANFIS classifier, which utilizes marks from the past stage as its info. This robotized grouping model orders the phase of variation from the norm utilizing ground truth esteem. The proposed characterization display is tried with Substantial fetal test picture datasets and it is contrasted with existing calculations with demonstrating its adequacy in identifying the fetal anomalies.
\end{abstract}

\section{Keywords: Hybrid ANFIS classifier, ABC, Fetal Abnormalities}

\section{INTRODUCTION}

Picture characterization is an issue that uses picture handling, design acknowledgment and grouping techniques. Programmed therapeutic picture characterization is a dynamic region in picture grouping, and it is required to be more created later on. As a result of this reality, programmed finding can help pathologists by giving second feelings and lessening their workload.

This work surveys the utilization of the versatile neurofluffy derivation framework (ANFIS) as a classifier in medicinal picture arrangement amid the past and at display. ANFIS is a fluffy induction framework (FIS) actualized in the system of a versatile fluffy neural system. It joins the express information portrayal of a FIS with the learning intensity of counterfeit neural systems. The goal of ANFIS is to coordinate the best highlights of fluffy frameworks and neural systems. A concise correlation with different classifiers, principle points of interest and downsides of this classifier are researched.

Programmed indicative frameworks are a critical use of examination of database and example acknowledgment, going for helping doctors in stamping demonstrative choices. Computerized conclusion is particularly used to analyze the assortment of diseases.

Revised Manuscript Received on August 20, 2019.

* Correspondence Author

Nagu Malothu*, Associate Professor, Department of ECE , V.K.R, V.N.B \& A.G.K College of Engineering, Gudivada, Krishna Dist. A.P., India ,Email: mng100174@gmail.com

Dr. V.V.K.D.V. Prasad, Professor \& Head, Department of ECE, Gudlavalleru Engineering College, Gudlavalleru, Krishna .Dist., A.P., India, Email: varrevkdvp@ rediffmail.com
Due to this reality, malignancy is the second driving reason for death of the two people on the planet, and is required to end up the main source of death in the following couple of decades. Mechanized indicative frameworks have been connected to and are of enthusiasm for an assortment of restorative information, including therapeutic signs and medicinal pictures. Among the utilizations of the versatile neuro-fluffy induction framework (ANFIS) in therapeutic finding are a few tests that utilization medicinal flags as info information for diagnosing maladies, for example, diabetes infections, blood corrosiveness, valvular heart sicknesses, rheumated joint pain ailment, epileptic seizure, prostate tumor, microarray growth, for example, colon malignancy, leukemia disease and lymphoma growth, optic nerve illnesses, recognizing Doppler flag, investigation of inner carotid blood vessel Doppler signals, distinguishing electroencephalogram (EEG) signals and ophthalmic blood vessel issue.

Notwithstanding, this paper underscores the restorative finding from therapeutic pictures. Therapeutic picture grouping has three principle steps: pre-preparing, highlight extraction and order. The pre-preparing step is done to improve the restorative picture. After pre-handling, different calculations are utilized for picture division to set up a medicinal picture for extricating highlights that are bolstered into a named input vectors. In all applications in which ANFIS is connected as classifier, the information vector of the ANFIS display comprises of highlights that are separated from a sectioned picture. In these works, distinctive techniques are utilized for the division and highlight extraction steps. Division and highlight extraction calculations area of this paper investigated these methodologies. Despite the fact that this work accentuates on ANFIS as a classifier, what's more, it can be utilized for other diverse assignments like de-noising or division. This work is trailed by brief cases of these two assignments.

\section{A. DENOISING}

The majority of the pictures are influenced through commotions and relics caused by the different obtaining systems and, subsequently, a powerful strategy for denoising is vital for medicinal pictures, especially in figured tomography, which is a noteworthy and most broad methodology in therapeutic imaging. The Authors in make utilization of ANFIS for expelling distinctive kinds of commotion from pictures, for example, Gaussian clamor, dot commotion and motivation clamor. Thus, de noised and quality-upgraded pictures are gotten.

Picture denoising is the key issue in Image preparing. Wavelet gives the brilliant execution in field of picture denoising due to sparsity and multi determination structure. 
With the prevalence of Wavelet Transform throughout the previous two decades, a few calculations have been produced in wavelet area. The concentration was moved to Wavelet space from spatial and Fourier area. As far back as the Donoho's wavelet based thresholding approach there was flood in the picture denoising is being distributed. Despite the fact that his approach was not progressive, it didn't require following and relationship of the wavelet maxima and minima over the distinctive scales as proposed, in this manner there was recharged enthusiasm for wavelet approach since Donoho's shown a straightforward answer for troublesome issue space. Scientists distributed distinctive ways to deal with figure the reproduction parameters for wavelet coefficients. To accomplish ideal edge esteem, information versatile limit were presented. Considerable enhancements in perceptual quality could be acquired by interpretation invariant strategy in view of thresholding of an UN annihilated Wavelet change. Much exertion has been given to Bayesian denoising in wavelet area. Gaussian scale blends and concealed markov models have additionally turned out to be prevalent and more research is kept on being distributed and Independent part investigations (ICA) have been investigated in information versatile segments. Distinctive measurable models are engaged to show the factual properties of wavelet coefficients and its neighbors. In the probabilistic models for non-symmetrical wavelet coefficients are circulated. Denoising is likewise finished with $\mathrm{ABC}$. Picture Enhancement is a critical stage in relatively every picture preparing framework. It goes for enhancing both the visual and enlightening nature of mutilated pictures. Histogram Equalization (HE) systems are the most famous methodologies for picture upgrade for they prevail with regards to improving the picture and protecting its primary attributes. Be that as it may, utilizing comprehensive methodologies for histogram leveling is an algorithmically complex errand. These HE procedures likewise bomb in offering great improvement if not very great parameters are picked. Along these lines, new wise methodologies, utilizing Artificial Intelligence procedures, have been proposed for picture improvement. In this specific situation, this paper proposes another Artificial Bee Colony (ABC) calculation for picture differentiate improvement. A dim level mapping system and another picture quality measure are utilized. The calculation has been tried on some test pictures, and the examinations of the got results with the hereditary calculation have demonstrated its prevalence. Also, the proposed calculation has been stretched out to shading picture improvement and given extremely encouraging outcomes. Promote subjective and measurable correlations of the proposed ABC to the Cuckoo Search (CS) calculation are likewise introduced in the paper; not just for the embraced dark level mapping method, yet in addition with utilizing another basic change, by and large called the neighborhood worldwide change.

\section{B. SEGMENTATION}

Picture division and its execution assessment are imperative viewpoints in picture preparing and, on account of the many-sided quality of the therapeutic pictures, division of restorative symbolism remains a testing issue. The ANFIS model can be utilized as an approach for the division of various ailments, for example, bosom growth, renal calculi and, particularly, mind tumor, fetal variations from the norm in first-trimester pregnancy meaning. The format of this work is as per the following: ANFIS segment presents the ANFIS structure and its learning calculation. In the division and highlight extraction calculations segment, the techniques for division and highlight extraction in medicinal picture applications in which the ANFIS display is connected as classifier are checked on. ANFIS classifier segment displays a study of works that utilized the ANFIS demonstrate as a classifier in medicinal picture order and a concise correlation with different classifiers is proposed. Likewise, the primary points of interest and downsides of ANFIS are examined in this segment. With a specific end goal to clear the point of this investigation, the structure of medicinal finding by utilizing the ANFIS display is introduced in Figure beneath The fundamental motivation behind this paper can be trailed by the strong line in the figure. At last, the paper deduces in the conclusion area.

Recognition and division of fetal unusual identification are imperative since it gives anatomical data on ordinary and anomalous tissues which helps in treatment arranging and patient development. There are various procedures for picture division. Proposed look into work utilizes ANFIS (Artificial Neural Network Fuzzy Inference System) for picture grouping and after that contrasts the outcomes and FCM (Fuzzy C means) and K-NN (K-closest neighbor). ANFIS incorporates advantages of both ANN and the fluffy rationale frameworks.

A far reaching highlight set and fluffy tenets are chosen to group an irregular picture to the relating tumor compose. Test results delineate promising outcomes as far as order exactness. A near examination is performed with the FCM and $\mathrm{K}-\mathrm{NN}$ to demonstrate the predominant idea of ANFIS frameworks.

\section{EXISTED METHOD}

The utilization of Artificial Bee Colony (ABC) calculation is to register pixel grouping for picture division. $\mathrm{ABC}$ is a heuristic calculation roused by the shrewd conduct of bumble bees which has been effectively utilized to take care of complex improvement issues. In this approach, a picture 1-D histogram is approximated through a Gaussian blend show whose parameters are ascertained by the ABC calculation. For the estimate conspire, each Gaussian capacity speaks to a pixel class and along these lines an edge. Dissimilar to the Expectation-Maximization (EM) calculation, the ABC-based strategy indicates quick union and low affectability to beginning conditions. Amazingly, it additionally enhances complex tedious calculations regularly required by slope based strategies. Trial results exhibit the calculation's capacity to perform programmed multi-edge choice yet demonstrating fascinating preferences by correlation with other understood calculations.

A few picture preparing applications plan to recognize and group pertinent highlights which might be later broke down to play out a few abnormal state assignments. Specifically, picture division looks to amass pixels inside significant districts. Generally, dim levels having a place with the question are significantly not the same as those highlighting on the foundation. Thresholding is in this way a basic yet viable device to disconnect objects of intrigue; its 
applications incorporate a few works of art, for example, report picture investigation whose objective is to separate printed characters, logos, graphical substance, or melodic scores; likewise it is utilized for delineate which means to find lines, legends, and characters. In addition, it is utilized for scene preparing, looking for protest location, stamping and for quality investigation of materials. Thresholding choice systems can be arranged into two classifications: bi-level and staggered. In the previous, one point of confinement esteem is portioned a picture into two classes: one speaking to the protest and the other one dividing the foundation. At the point when unmistakable articles are portrayed inside a given scene, various edge esteems must be chosen for legitimate division, which is normally called staggered thresholding. Despite the fact that an assortment of thresholding approaches have been proposed for picture division, including traditional strategies and canny procedures, stretching out the division calculations to a staggered approach may cause a few burdens: (I) they may have no efficient or explanatory arrangement when the quantity of classes to be identified increments and (ii) they may likewise demonstrate a moderate meeting or potentially high computational cost. In this section, the division calculation depends on a parametric model that holds a likelihood thickness capacity of dim levels which bunches a blend of a few other Gaussian thickness capacities (Gaussian blend). Blends speak to an adaptable technique for factual displaying as they are utilized in a wide assortment of settings. Gaussian blend has gotten extensive consideration in the improvement of division calculations regardless of its execution is affected by the state of the picture histogram and the exactness of the evaluated show parameters . The related parameters can be computed thinking about the Expectation Maximization (EM) calculation or Gradient-based strategies, for example, Levenberg-Marquardt, LM. Be that as it may, EM calculations are exceptionally delicate to the decision of the underlying qualities, in the interim Gradient-based techniques are computationally costly and may effortlessly stall out inside nearby minima. In this manner, a few analysts have endeavored to create strategies in view of present day worldwide advancement calculations, for example, the Learning Automata (LA) and the differential development calculation. In this section, an elective approach utilizing an enhancement calculation for deciding the parameters of a Gaussian blend is displayed. Then again, the social creature allegory for taking care of issues has turned into an intriguing subject in the most recent years, specifically the social bug illustration. This approach stresses a circulated method for working with associations among operators that could be considered as straightforward, strong and adaptable.

This has introduced a meta-heuristic calculation for taking care of numerical enhancement issues known as the fake honey bee settlement (ABC) technique. Enlivened by the keen scavenging conduct of a bumble bee swarm, the $\mathrm{ABC}$ calculation comprises of three basic parts: nourishment source positions, nectar-sums and a few bumble bee classes. Each source position speaks to a practical answer for the issue under thought.

The nectar-sum for a sustenance source speaks to the nature of such arrangement as indicated by its wellness esteem. Every honey bee class symbolizes one specific activity for producing new applicant source positions (i.e. applicant arrangements). The order of MR pictures is ending up progressively imperative in the restorative field since it is

significant for treatment arranging and diagnosing variation from the norm (For e.g fetal image),measure tissue volume to see irregular development, think about anatomical structure and patient development. Manual characterization of attractive reverberation (MR) strange fetal pictures is a testing and tedious errand. Manual arrangement is profoundly inclined to mistake due to entomb onlooker fluctuation and human blunder. Thus, the characterization results are profoundly second rate which prompts deadly outcomes. Along these lines, a programmed or self-loader characterization technique is very attractive as it lessens the heap on the human spectator, extensive number of cases can be dealt with same precision, additionally, results are not influenced because of weariness, information over-burden, quicker correspondence. There are no all inclusive calculation for division of each restorative pictures. Distinctive body parts MRI picture needs unique sort of division. Different strategies proposed in the writing have met with just constrained accomplishment because of covering force circulations of sound tissue, fetal, and encompassing edema. The most well-known class of strategies is factual grouping utilizing multi parameter pictures. These strategies are exceptionally power based and subsequently the precision is low. Another gathering of scientists profoundly rely upon computational knowledge for fetal strange order which ensures high precision. The Self Organizing Feature Map (SOFM) ANN based calculations demonstrates superb outcomes in the arrangement of trimester pictures. Different investigations in light of learning vector quantization (LVQ) ANN demonstrate the capability of these structures on account of regulated grouping. Hopfield neural systems (HNN) end up being productive for unsupervised example characterization of medicinal pictures, especially in the identification of unusual tissues. The utilization of ART2 organize for design acknowledgment has been considered by Solis and Perez. A few alterations on the current neural systems are actualized effectively and unrivaled outcomes have been accomplished. Other than being powerful, they require substantial preparing dataset to accomplish high exactness. This builds the dimensionality issue which represents the many-sided quality of the model. Then again, a few inquires about in view of fluffy rationale procedures are additionally detailed in the writing. These utilized administer based fluffy rationale derivation for MR cerebrum picture order. Analyses in light of fluffy $\mathrm{C}$-implies calculation are likewise proposed in the writing is executed an altered fluffy $\mathrm{C}$ implies calculation for picture order. The fluffy set theoretic models endeavor to impersonate human thinking and the capacity of taking care of vulnerability, while the neural system models endeavor to copy the engineering and data portrayal plans of the human cerebrum. Mix of the benefits of the fluffy set hypothesis and neural system hypothesis guarantees to give, all things considered, more wise frameworks to deal with genuine issues. A neuro-fluffy approach as a blend of neural systems and fluffy rationale has been acquainted with defeated the individual shortcomings and to offer all the more engaging highlights. 


\section{PROPOSED WORK}

\section{A. AABC method}

In our propose calculation, we utilize adjusted streamlining idea with ABC-PSO and document technique to influence the calculation to meet to the PSO and GA. The best favorable position of ABC-PSO is that it could utilize less control parameters to get the most focused execution. Keeping in mind the end goal to show the execution of the ABC-PSO calculation, we thought about the execution of the ABC-PSO calculation with that of GA and PSO improvement calculations on a few two-target test issues and three-target capacities.

\section{B. AABC Algorithem}

1: Initialization phase; Initialization parameters

2: PSO optimization: /*Use PSO neighborhood formula for getting best fitness solution*/ a) For each parameter:

\subsection{Calculate the fitness value}

2.2 If the fitness value is better than the best fitness value (Pbest) in history

2.3 Set current value as the new Pbest /*getting best fitness value.*/ End b) For each parameter:

2.4 Find in the particle neighborhood, the particle with the best fitness.

2.5 Calculate particle velocity according to the velocity equation.
2.6 Apply the velocity constriction. End While until a termination criterion is met. /*After that go to the next step.*/

3: Onlooker bee phase.

3.1 Assign the best Onlooker bee to the Employed bee accordingly to the Pbest value.

3.2 For all Onlooker bees.

\subsection{Ejection- chain Neighborhood.}

3.4 Find the best onlooker, replace with respect to Pbest Neighborhood value. If fit (Best Onlooker) <

p(best) 3.5 Find best Feasible Onlooker, replace with Best solution, If fit (Best Feas Onlooker) < (best)

$3.6 \mathrm{~N}=\mathrm{N}+1$;

3.7 Until $(\mathrm{N}=$ pbest value $)$;

$3.8 \mathrm{I}=\mathrm{I}+1$

3.9 until I = Maximum Cycle Number Evaluate each particle in the size of home

3.10 Discover the best solution based on precision/recall and check for all the particles: if the Calculated value xi is not better than the new solution, then xi is replaced by the new solution

\section{A. proposed diagram of modified optimization technique by AABC}

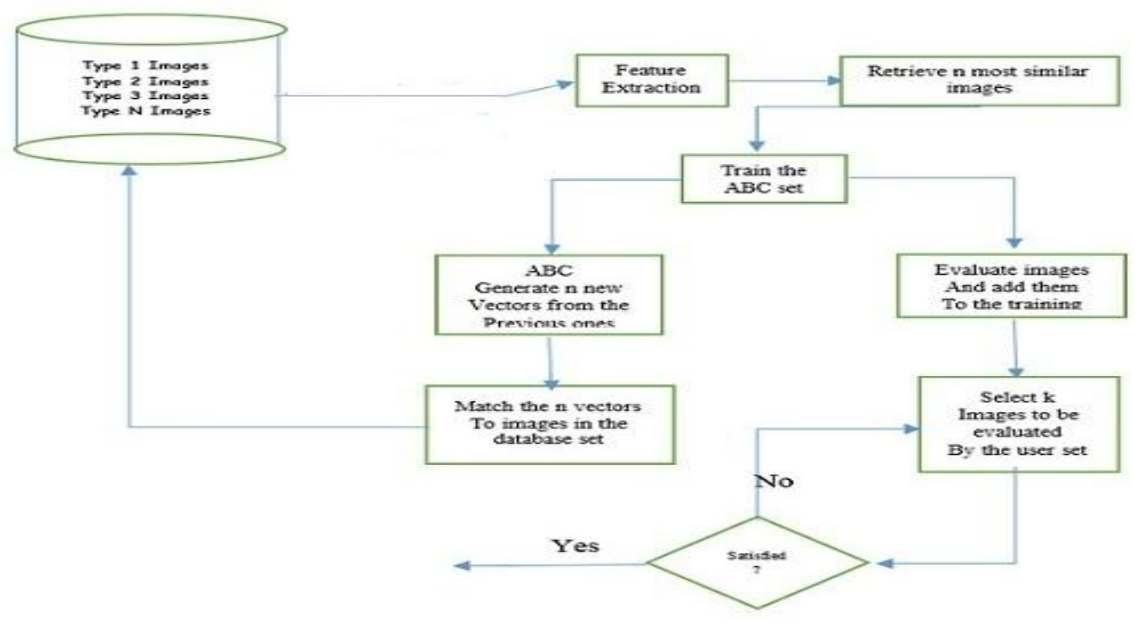

Fig.1. AABC method

Figure above clarifies that robotized fake honey bee settlement technique here AABC is connected on the chosen fetal strange picture to discover the issue in that picture plainly.

\section{B. Proposed AABC-ANFIS}




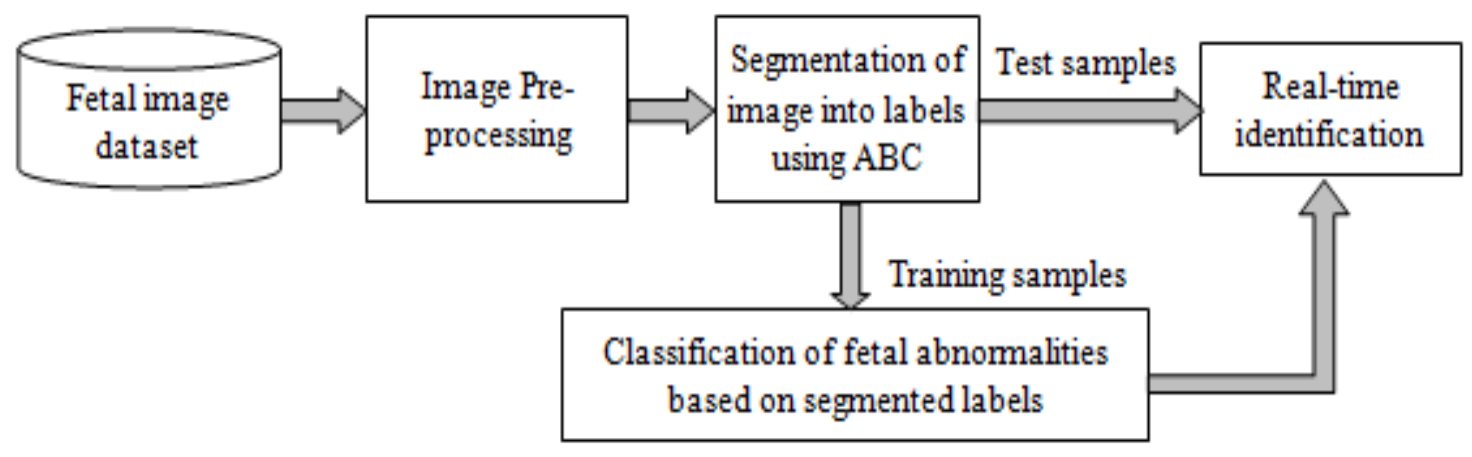

Fig.2. Proposed Method

Above figure clarifies that unmistakable program of our work first we are select the fetal picture from that picture remove information and from the informational index. In following stage apply picture preparing systems like division name level extraction by utilizing AABC-strategy. Encourage we isolated the examples into two kinds 1. Test tests 2. Preparing tests. At long last utilizing ANFIS rationale to separate the information and give the Problem unmistakably, Finally done all parameters.

\section{EXPERIMENTAL RESULTS}

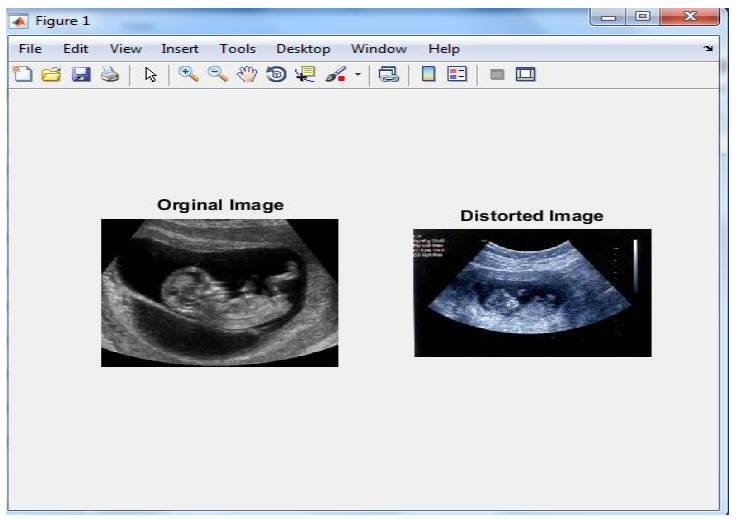

Fig. 3. AABC output Removing Noise

\section{Wavelet decomposed image with $\mathrm{AABC}$}

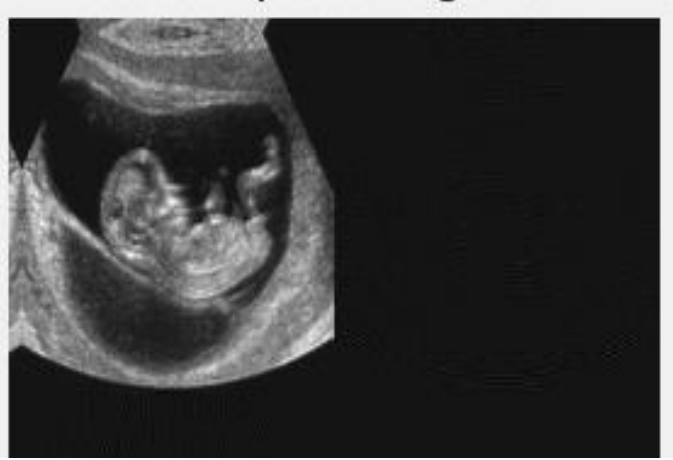

Fig.4. wavelet decomposition with AABC

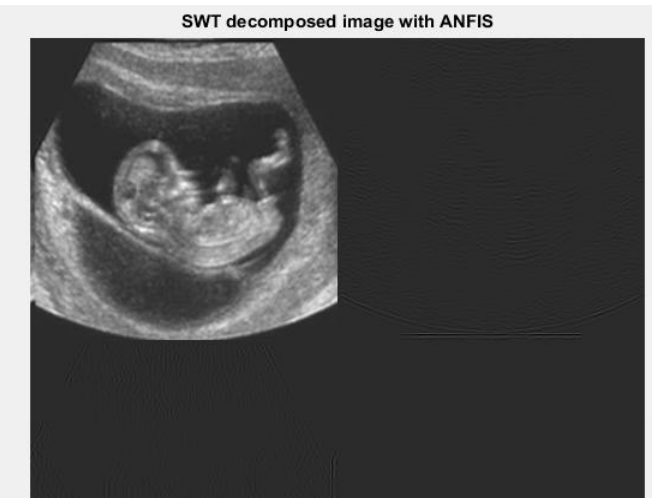

Fig.5. ANFIS based label extracted image

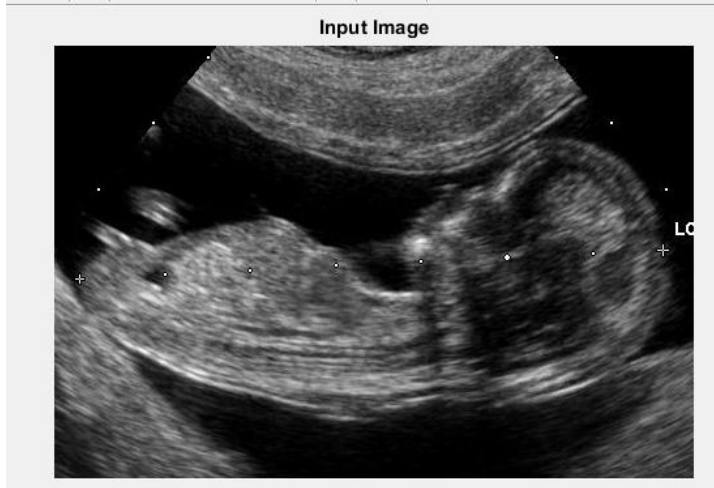

Fig.6. input image to proposed method

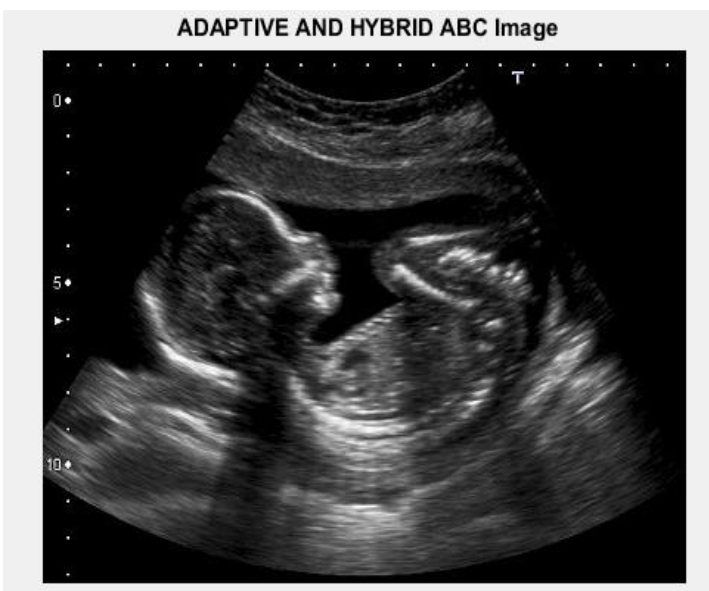

Fig.7. AABC-Hybrid segmentation Method Output

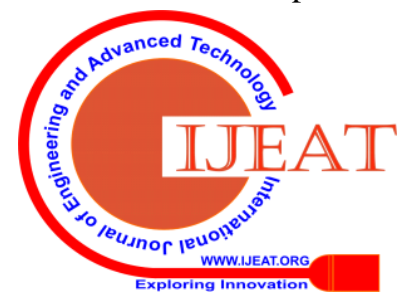




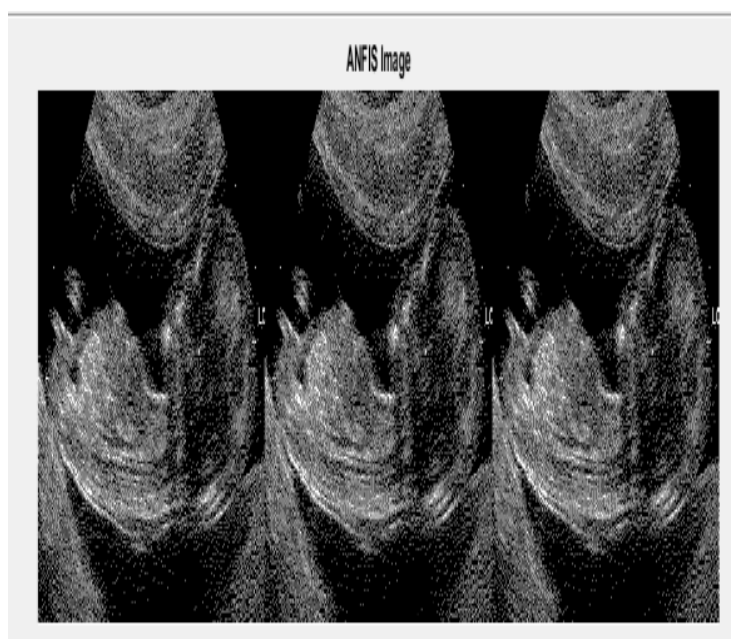

Fig.8. ANFIS-layered based image

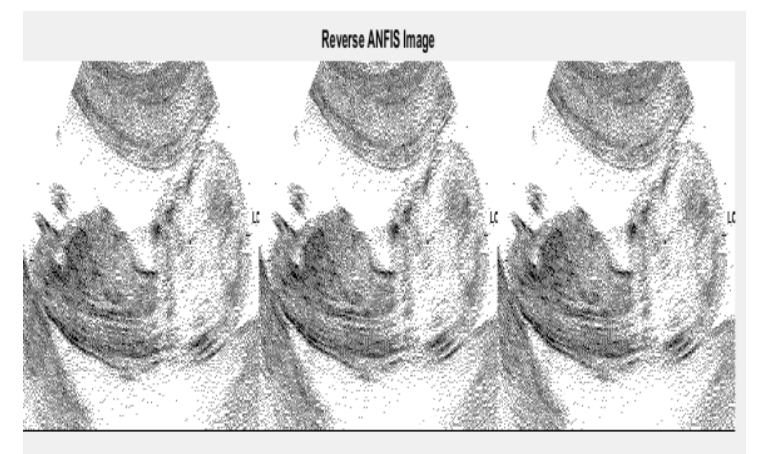

Fig.9. Final Extracted Image

Figure 3,4,5,6,7,8,9 explains that ouputs of AABC-ANFIS algorithm. Here we observed that segmented image and noise removable image and layered image.

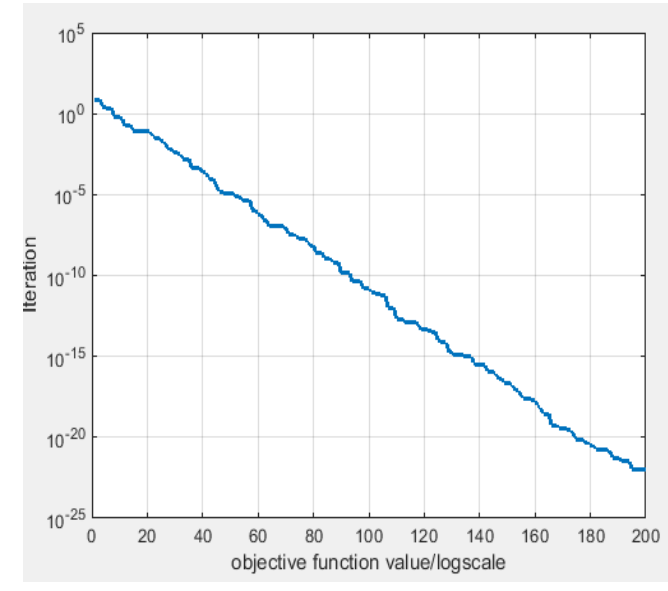

Fig.10. Iteration vs Object Function Value

\section{AABC-ANFIS DATA SET}

Iteration 189: objective function value $=5.4253 \mathrm{e}-22$

Iteration 190: objective function value $=5.4253 \mathrm{e}-22$

Iteration 191: objective function value $=3.9655 \mathrm{e}-22$

Iteration 192: objective function value $=3.4035 \mathrm{e}-22$

Iteration 193: objective function value $=3.4035 \mathrm{e}-22$

Iteration 194: objective function value $=3.4035 \mathrm{e}-22$
Iteration 195: objective function value $=1.089 \mathrm{e}-22$

Iteration 196: objective function value $=1.089 \mathrm{e}-22$

Iteration 197: objective function value $=1.089 \mathrm{e}-22$

Iteration 198: objective function value $=1.089 \mathrm{e}-22$

Iteration 199: objective function value $=1.089 \mathrm{e}-22$

Iteration 200: objective function value $=1.089 \mathrm{e}-22$

\section{Numerical Measure}

97.3418

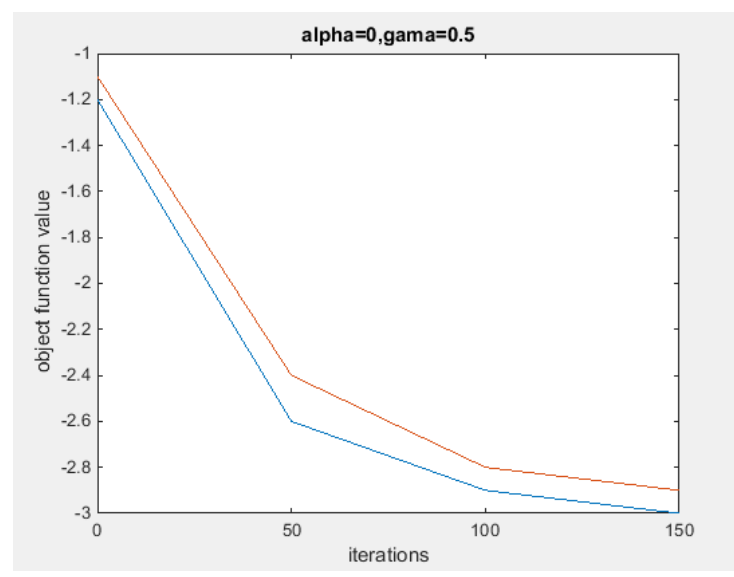

Fig. 11. Data Set Analysis

Fig 11 explains that data analysis at alpha $=0$ and gama $=0.5$

TABLE-1: Data Set Analysis

\begin{tabular}{|l|l|l|l|}
\hline \multicolumn{4}{|c|}{ AABC-ANFIS DATASET } \\
\hline No of rounds & precision & recall & f-measure \\
\hline 1 & 0.8 & 0.13 & 0.23 \\
\hline 2 & 0.57 & 0.35 & 0.43 \\
\hline 3 & 0.61 & 0.58 & 0.53 \\
\hline
\end{tabular}

Compared to past techniques this proposed algorithm gives the best outputs and efficiency

\section{CONCLUSION}

In this area the half and half AABC-ANFIS technique is presented by hybridization of AABC and PSO calculations. The proposed calculation is actualized in MATLAB and after that all around tried by various diverse standard assessment capacities. The parameter position is enhanced for various protests and the result of the proposed calculation is contrasted and single target GA, PSO and $\mathrm{ABC}$ calculations. In the proposed calculation, it is watched that the wellness esteems for exactness, review and Fmeasure is superior to lone assessment calculations. Based on the above results, it is clearly observed that the performance of AABC-ANFIS is the better than individual $\mathrm{GA}, \mathrm{PSO}$ and $\mathrm{ABC}$ algorithms.

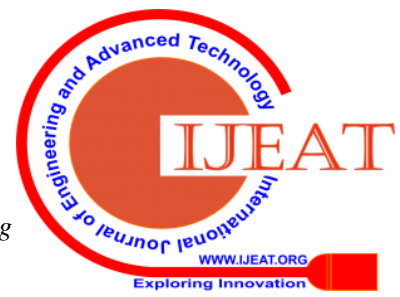




\section{REFERENCES}

1. J.P.S. Catalão, H.M.I. Pousinho, V.M.F. Mendes, Hybrid WaveletPSO-ANFISapproach for short-term electricity prices forecasting, IEEE Trans. Power Syst.26 (1) (2011) 137-144.

2. A. Chatterjee, K. Watanabe, An optimized Takagi-Sugeno type neuro-fuzzysystem for modeling robot manipulators, Neural Comput. Appl. 15 (1) (2006)55-61.

3. A. Sarkheyli, A.M. Zain, S. Sharif, Robust optimization of ANFIS based on a new modified GA, Neuro computing 166 (2015) $357-$ 366.

4. L.Y. Wei, A GA-weighted ANFIS model based on multiple stock market volatility causality for TAIEX forecasting, Appl. Soft Comput. 13 (2) (2013)911-920.

5. P. Liu, W. Leng, W. Fang, Training ANFIS model with an improved quantum-behaved particle swarm optimization algorithm, Math Probl. Eng.2013 (2013) (2013).

6. J.P.S. Catalão, H.M.I. Pousinho, V.M.F. Mendes, Hybrid WaveletPSO-ANFIS approach for short-term electricity prices forecasting, IEEE Trans. Power Syst.26 (1) (2011) 137-144.

7. S. Suja Priyadharsini, S. Edward Rajan, S. Femilin Sheniha, A novel approachfor the elimination of artefacts from EEG signals employing an improvedArtificial Immune System algorithm, J. Exp. Theor. Artif. Intell. (2015) 1-21.

8. M. Gunasekaran, K.S. Ramaswami, A fusion model integrating ANFIS andartificial immune algorithm for forecasting indian stock market, J. Appl. Sci.11 (16) (2011) 3028-3033.

9. M. Asadollahi-Baboli, In silico prediction of the aniline derivatives toxicitiesto Tetrahymena pyriformis using chemometrics tools, Toxicol. Environ. Chem.94 (10) (2012) 2019-2034.

10. R. Teimouri, H. Baseri, Optimization of magnetic field assisted EDM using the continuous ACO algorithm, Appl. Soft Comput. 14 (2014) 381-389.

11. Tao Xiang a, Kwok wo Wong, and Xiaofeng Liao. A novel particle swarm optimizer with time-delay. Applied Mathematics and Computation, 186:789-793, 2007.

12. A. Ahmadi, F. Karray, and M. Kamel. Multiple co-operating swarms for dataclustering. In Swarm Intelligence Symposium, pages 206-212. IEEE, 2005.

13. Madhabananda Das A.K Mishra and T.C Panda. A hybrid swarm intelligence optimization for benchmark models by blending pso with abc. International Review on Modelling and Simulations, 6(1):291, 2013

14. Y. Tan andM. Xiao. Clonal particle swarm optimization and its applications. In IEEE Congress onEvolutionary Computation,CEC 2007, pages 2303-2309. IEEE, september 2007.

15. Inthachot $\mathrm{M}$ andSupratid $\mathrm{S}$. A multi-subpopulation particle swarm optimization: A hybrid intelligent computing for function optimization. In Third International Conference on Natural Computation, ICNC 2007, volume 5, pages 679-684. IEEE, august 2007.

16. M. Senthil Arumugam, M.V.C. Rao, and Alan W.C. Tan. A novel and effective particle swarm optimization like algorithm with extrapolation technique. Applied Soft Computing, 9:308-320, 2009.

17. ArunKhosla, Shakti Kumar, and K.K. Aggarwal. Identification of strategy parameters for particle swarm optimizer through taguchi method. Journal of Zhejiang University SCIENCE A, 7(12):19891994, 2006

\section{AUTHORS PROFILE}

NAGU MALOTHU, working as an Associate Professor in ECE Department in V.K.R, V.N.B \& A.G.K college of engineering, Gudivada. $\mathrm{He}$ did his B.Tech in MJ College of engineering and technology, Hyderabad and M.Tech from JNTUH, Hyderabad. He has 18 years of teaching experience and 12 publications to his credit. Currently pursuing Ph.D at JNTUK, Kakinada.

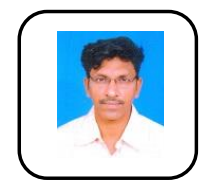

Dr. V. V. K. D. V. Prasad, working as a Professor and Head of the Department, of Electronics \& Communication Engineering, in Gudlavalleru Engineering College, an Autonomous NBA accredited College in Andhra Pradesh, India. He received Ph.D for his work in Signal Processing; in 2011 from Jawaharlal Nehru technological University, Kakinada, India. His areas of Interest include Signal Processing, Electrostatics, Electromagnetic fields and Transmission lines. He developed an abstract technical trait that addresses various other fields where digitalization can be achieved. His research findings are in the methodology used, problems encountered and thepractical implications of composite features and filtering coefficients in advanced filtersMr. Rohith Bala Jaswanth B is a Research Scholar, pursuing Ph.D from Jawaharlal Nehru Technological University, Kakinada, and Andhra Pradesh, India. He has been working as an Academician in the capacity of An Assistant Professor in Gudlavalleru Engineering College, a reputed Autonomous Engineering College in Andhra Pradesh, India. Apart from regular Academia, the zeal to explore new technologies and a penchant towards pursuing research in the field of Analog \& Digital Electronics has made him to do the research work in a governmental organization in Andhra Pradesh. His keen interests are inclined more towards devices and technologies of Analog \& Digital IC design. His areas of Interest include Low Power VLSI, Probability Theory and Estimation, Pulse \& Digital Circuits, Biomedical Engineering, Linear \& Digital ICS and Applications. profile which contains their education details, their publications, research work, membership, achievements, with photo that will be maximum 200-400 words. 